ÁREA ABIERTA. Vol.14. $n^{\circ}$ 1. Marzo 2014

http://dx.doi.org/10.5209/rev_ARAB.2014.v35.n1.44687

“ANÁLISIS LINGÜÍSTICO-SEMIÓTICO. IDENTIDAD DE MARCA.

VALORIZACIÓN UTÓPICA"

AUTORA: Lidia PELLICER GARCÍA

Universidad Católica San Antonio de Murcia, España

\title{
Análisis lingüístico-semiótico. Identidad de marca. Valorización utópica
}

\author{
Linguistics-semiotic Analysis. \\ Identity Brand: Utopian Value
}




\section{RESUMEN}

Desde la lingüística los nombres propios no tienen significado aduciéndose que lo peculiar es su ausencia de rasgos sémicos preexistentes, sin valor conceptual. El objetivo de este trabajo es demostrar que en publicidad el nombre propio es una marca con significado, un signo. Para ello, nuestra metodología consistirá en el análisis de dos spots publicitarios de la marca Movistar que nos llevará a la conclusión de que la marca publicitaria es un signo portador de significado identitario.

\section{Palabras clave}

Nombre propio, Significado, Signo, Publicidad.

\section{ABSTRACT}

Of a Linguistic point of view, the proper names there aren't meaning because his characteristic is the signifier absence without conceptual value. Present work's objective is proved in advertising the proper noun means, is a sign. For this, our methodology is the two Movistar brand's advertising commercial analysis that comes us to the conclusion to the brand is a sign with identity and meaning.

\section{Key words}

Proper name, Meaning, Sign, Advertising. 


\section{INTRODUCCIÓN}

"Las firmas comerciales se lanzan a la conquista del mercado mediante el impacto que produce la presencia insistente del nombre propio del producto en los medios de comunicación de masas", afirma Sánchez Corral (1990: 207). La creación de la marca supone, pues, uno de los objetivos de carácter fundamental para las empresas. En su creación y gestión cooperan diferentes aproximaciones (económica, psicológica, informativa, social, marketing ${ }^{1}$ ), dentro de las cuales se encuentra la perspectiva lingüística centrada en aspectos fonéticos, morfológicos y semánticos.

En este artículo vamos a detenernos en la aproximación lingüística desde el punto de vista semiótico en aras de estudiar la gestión del significado identitario de la marca española Movistar de la compañía Telefónica. Para ello hemos seleccionado un spot de televisión realizado con motivo de la campaña de lanzamiento de Movistar en $1996^{2}$ que nos llevará a la conclusión de que el nombre propio Movistar es una marca con significado, frente a los postulados de las teorías lingüísticas para las que el nombre propio carece de significado ${ }^{3}$, mediante el cual el sujeto realiza su propia identidad conjugándose con su objeto de valor.

\footnotetext{
${ }^{1}$ DEL RÍO LANZA, A.; VÁZQUEZ CASIELLES, R.; IGLESIAS ARGÜELLES, V. (2002): <<El valor de marca: perspectivas de análisis y criterios de estimación>>, Cuadernos de gestión, 1, Madrid, Universidad Complutense de Madrid, 87-102.

${ }^{2}$ Pérez Yuste (2002: 26-31) realiza un estudio sobre el proceso de la implantación de la telefonía móvil en España y señala al respecto de estos años que vamos a estudiar en este artículo que terminadas las Elecciones Generales de marzo de 1996, comienza un trienio de actuaciones conducentes a la plena liberalización de las infraestructuras y de los servicios de telecomunicación eligiéndose (por parte del Gobierno Popular) el "duopolio" basado en dos empresas públicas: Telefónica y Retevisión, su posterior privatización y, finalmente, la apertura total del mercado el 1 de diciembre de 1998.

Tras cambiar el Gobierno, se creó un segundo operador global de telecomunicaciones, el 7 de junio de 1996 , otorgando a la empresa pública Retevisión un título habilitado para el servicio de telefonía básica así como para el servicio portador.

En ese mismo año se creó también la Comisión del Mercado de las Telecomunicaciones como órgano gubernamental independiente encargado de velar por las condiciones de competencia efectiva en el mercado, por la correcta formación de los precios y ejercer de órgano arbitral en los conflictos que surgiesen en el sector.

Una vez establecido el "duopolio" el siguiente paso fue iniciar el proceso de adjudicación de activos de ambas compañías. De esta forma, el 18 de enero de 1997 se puso a la venta el 20, 9 \% de Telefónica, que estaba en manos del Estado, mediante la Oferta Pública de Venta (OPV) más publicitada de todos los tiempos (en 1994 Telefónica ya se había privatizado en un 12\%) dejando de ser una compañía pública en el plazo de un mes, igual que le sucedería a Retevisión el 11 de julio de 1997 pasando al consorcio formado por el operador italiano STET (Telecom Italia) y las Eléctricas Endesa y Unión FENOSA.

${ }^{3}$ Como señala García Gallarín (García Gallarín, 1999, p. 19), con los estudios de Mill (1843) sobre el sentido del nombre propio comienza un debate que todavía persiste en la actualidad acerca del significado del nombre propio; dicho debate introdujo la aparición de las dos teorías más relevantes sobre la referencia del nombre propio: la histórico-causal, cuyo precursor es Kripke (1971), según la cual el nombre propio carece de significado y designa a un referente único en virtud de una cadena causativa siendo su origen un acto de bautismo; y la descriptiva, defendida sobre todo por Frege (1976), Russell (1903), Searle (1979) y Strawson (1971), centrada en la existencia del nombre propio en el discurso donde éste tiene sentido porque identifica de manera unívoca al referente.
} 


\section{EL VALOR UTÓPICO GENERADOR DE IDENTIDAD DISCURSIVA EN EL OBJETO MÓVIL}

A partir de las valorizaciones que Floch establece en su cuadrado semiótico de la axiología del consumo (valorización práctica, utópica, lúdica y crítica), se redefine el valor de uso como valor práctico y el de base como valor utópico; valores que, junto con los otros dos; el crítico y el lúdico, Marrone (2002: 17) define de la siguiente manera:

-Valorización práctica. "Una valorizzazione pratica, con la quale si considera l'oggetto sopratutto per il suo carattere di strumento".

-Valorización utópica. "Una valorizzazione utópica, mediante la quale il soggetto realiza la propia identitá congiungendosi con il suo oggetto di valore".

-Valorización lúdico-estética. "Una valorizzazione ludico-estetica corrispondente alla negazione di quella pratica, dove si considera l'oggetto, prescindendo dalla sua utilizzabilitá, a partire dalle sue qualitá formali e fisiche, del piacere che procura, dalla sua belleza".

-Valorización crítica. Una valorización en la que "I'oggetto viene scelto più che altro per la sua convenienza".

En el presente artículo nos encontramos con una valorización utópica donde el sujeto realiza su propia identidad cuando se fusiona con su objeto de valor, el cual pasa de ser un objeto a un sujeto en su discursividad mediante estas valorizaciones que desencadenan pasiones, como la del deseo en este caso (Marrone, 1999).

El objeto producto se reviste de personalidad adquiriendo su propio carácter. En esta etapa de Movistar un rasgo de personalidad es la autorrealización íntima donde el sujeto se realiza personal y afectivamente, mostrando, de esta forma, un discurso donde la acción se torna pasión.

A este respecto, Fabbri estudia el problema de la concatenación de la acción y la pasión, destacando cómo ya Barthes (1973) se escindía entre desarrollar una semiótica rigurosa por un lado, y por otro, dar cuenta de la afectividad, mostrando una preocupación por la problemática de la afectividad como parte del proceso de significación del signo, expresa Fabbri (1998: 34-35):

"Roland Barthes ricorda che uno de suoi grandi problemi era quello di mantenere, da una parte, una teoria semiotica rigorosa e, dall'altra parte, la profonda esigenza di dar conto dell'affectivitá. Data l'imposibilitá di coniugare insieme le due cose, l'affectivitá finiva in Barthes per trovare espressioni differenti rispetto al modello che la sua semiologia proponeva. Da qui l'insistenza sulla necesita 
di far entrare all'interno della problemática del segno la questione dell'affectivitá."

De esta forma, Fabbri (1998: 35) señala dicho libro de Barthes como la primera obra en la que se articula el afecto unido a la problemática de la significación. No obstante, Fabbri apunta en la siguiente cita (1998: 35) que Barthes influenciado por las ideas de Lacan, quien a su vez partía de Jakobson y de Lévi- Strauss, asoció afectividad a psicoanálisis:

\begin{abstract}
"L'estensione delle riflessioni jakobsoniana e levistraussiana alla psicoanalisi era pensata in quegli anni come una naturale maniera di riformaulare la talking cure, la cura parlante che si otteneva in via puramente lingüística. Cosí, in qualche misura la psicoanalisi in quel periodo, come dicono i francesi, "prendeva lingua" con la semiotica e con la lingüística, sulla base della linguisticitá caratterizzante la cura psicoanalitica."
\end{abstract}

Fabbri (1998: 37), escindiendo la pasión de la razón, propone un análisis de la pasión en el que "la semiotica deve pensare i segni come azioni, come trasformatori di situación, come messa in campo e modificazione di attori, spazi e tempi".

Partiendo de estos razonamientos y siguiendo los postulados de Fabbri quien une los dos tratamientos aristotélicos presentes en la Retórica, argumentación y pasión, nos planteamos la siguiente pregunta: ¿ Qué tipo de acciones y de razones provocan en esta etapa el desarrollo de la pasión?

Para responder a esta pregunta nos centraremos en un componente señalado por Fabbri : el componente estésico.

\title{
1.1. El componente estésico
}

¿Cómo tiene lugar la transformación de la pasión a través de la acción en la dimensión sensorial? Como señala Fabbri (1998: 42), "Lo studio della dimensione passionale ci habitúa a un'idea ovvia: non c'é passione senza corpo".

Por tanto, siendo el discurso de marca especialmente estésico en esta etapa de su vida, vamos a analizar la configuración de la sensación expresada somáticamente a través del cuerpo asociada a una semántica discursiva.

¿Qué sensaciones despliega el discurso de marca a través de las acciones somáticas de los sujetos?

La sensación transmitida y deseada por los sujetos del relato es la del contacto, la cual constituye una constante en la vida del móvil pero cuya representación varía en su 
diacronía. ¿ Cómo se representa este deseo somáticamente? A través de la mirada, y el tacto.

\subsubsection{La mirada}

Mediante la mirada se pueden expresar diversas sensaciones, en este caso nos encontramos con personajes cuya mirada está focalizada hacia una realidad que el coenunciatario desconoce. La mirada transmite diferentes emociones según el personaje en los spots seleccionados. Con todo, podríamos establecer la siguiente caracterización del mirar:

- Una mirada atenta, profunda y seria

- Una mirada enfadada

- Una mirada apelativa

- Una mirada sensual

- Una mirada pensativa

- Una mirada feliz

La acción de mirar sustituye cualquier enunciación lingüística en los sujetos en esta parte del relato: su mirada es suficiente para comunicar lo que están sintiendo en ese preciso momento. Acción que aparece en primer plano en la mayoría de los casos.

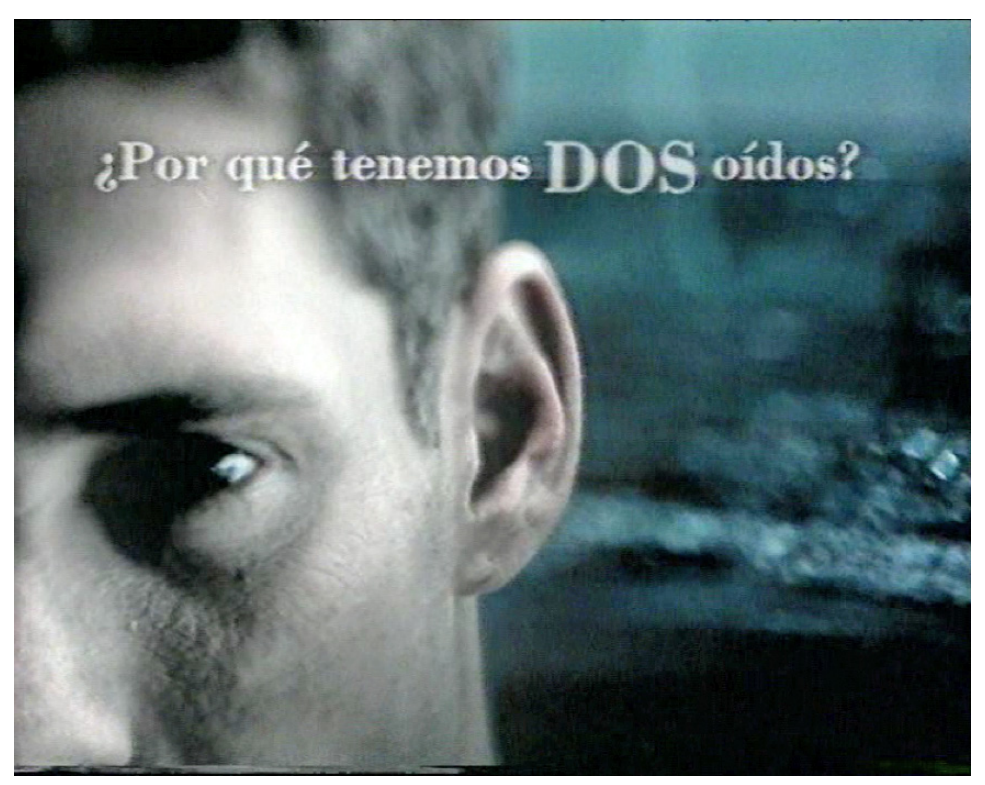

Fig. 1. Componente estésico. La mirada. Movistar (Telefónica, 1996) 


\subsubsection{El tacto}

Junto a la acción de mirar, el sentido del tacto constituye otra de las manifestaciones visuales más representadas en el relato. Dicho sentido lo encontramos ilustrado de varias formas:

1) En primer lugar, a través de las manos. Nos encontramos en el spot con la focalización en primer plano, ocupando el lateral izquierdo de la escena, de dos manos en movimiento en una relación de proximidad totalmente abiertas.

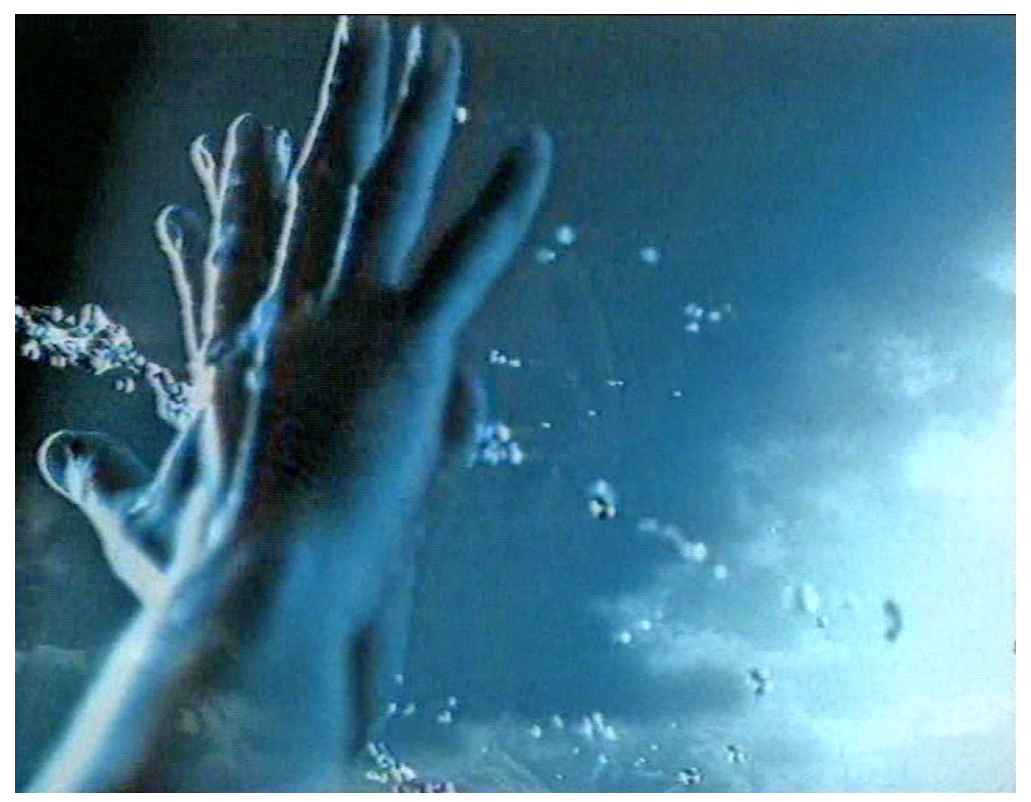

Fig. $n^{\circ}$ 2. Componente estésico. El tacto. Movistar (Telefónica, 1996)

Posteriormente aparece la representación de la acción de las manos en la imagen de la primera pareja del spot. La visualización de esta pareja permite observar cómo el hombre apoya su mano sobre el hombro de la preocupada mujer.

De nuevo, la caricia aparece figurativizada en escena en otro sujeto masculino, nos estamos refiriendo a la imagen de la segunda pareja que encontramos en el spot, donde el sujeto masculino acaricia con su mano el vientre de la actante femenina que muestra sus manos abiertas y extendidas sobre la arena.

A continuación, una niña acaricia un conejo cariñosamente y con mucho cuidado.

La apertura de las manos la encontramos, también, en la imagen de la chica en el borde de un acantilado. 
2) En segundo lugar, a través de los pies. Este miembro del cuerpo también tiene su actuación en el relato de la siguiente forma:

Para empezar, nos encontramos con la imagen de unos pies en contacto acariciándose extendidos en la arena.

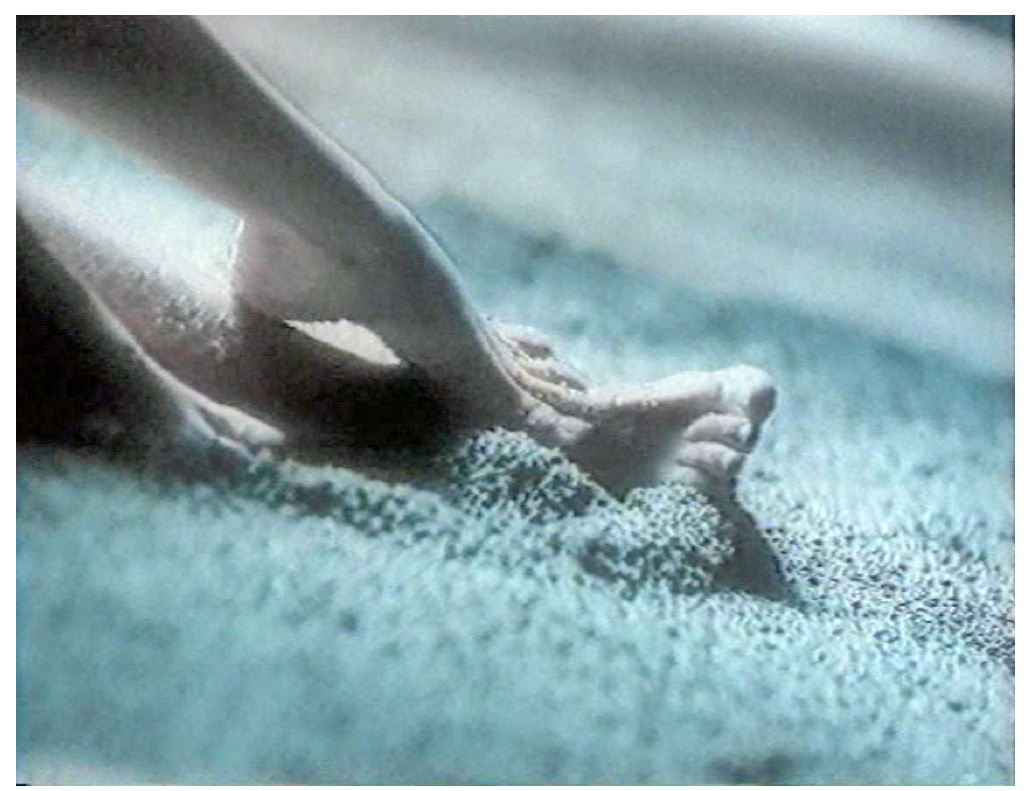

Fig. $n^{\circ}$ 3. Componente estésico. El tacto. Movistar (Telefónica, 1996)

A continuación la actuación del pie permite a los personajes el movimiento, representado en la acción de correr y de pasear.

¿Por dónde se mueven estos personajes? El deseo de contacto muestra a unos personajes únicos en lugares solitarios siendo el espacio escogido para la representación de la soledad la playa y el interior del hogar. Estamos ante la constante dicotómica exterior/interior. No obstante, el espacio más representado es el exterior en el que el elemento natural del agua vertebra la estructura discursiva. Sin duda, la apertura que caracteriza este discurso se extiende al espacio en el que se realizan los sujetos.

3) Por último, a través de la boca cuya acción la encontramos representada, por un lado, en la imagen de una niña que está gritando "en silencio", puesto que el coenunciatario no accede al sonido lingüístico de los sujetos de la escena.

Todas estas acciones somáticas manifiestan diferentes sensaciones según el sujeto del relato, ya que cuando éste se presenta solo en escena, las sensaciones transmitidas son las de la tristeza, la reflexión, el reclamo de atención y la soledad; mientras que cuando el sujeto aparece conjugado con su objeto de valor (con su signo), los síntomas somáticos reflejan alegría, amor, sensualidad y cariño. 


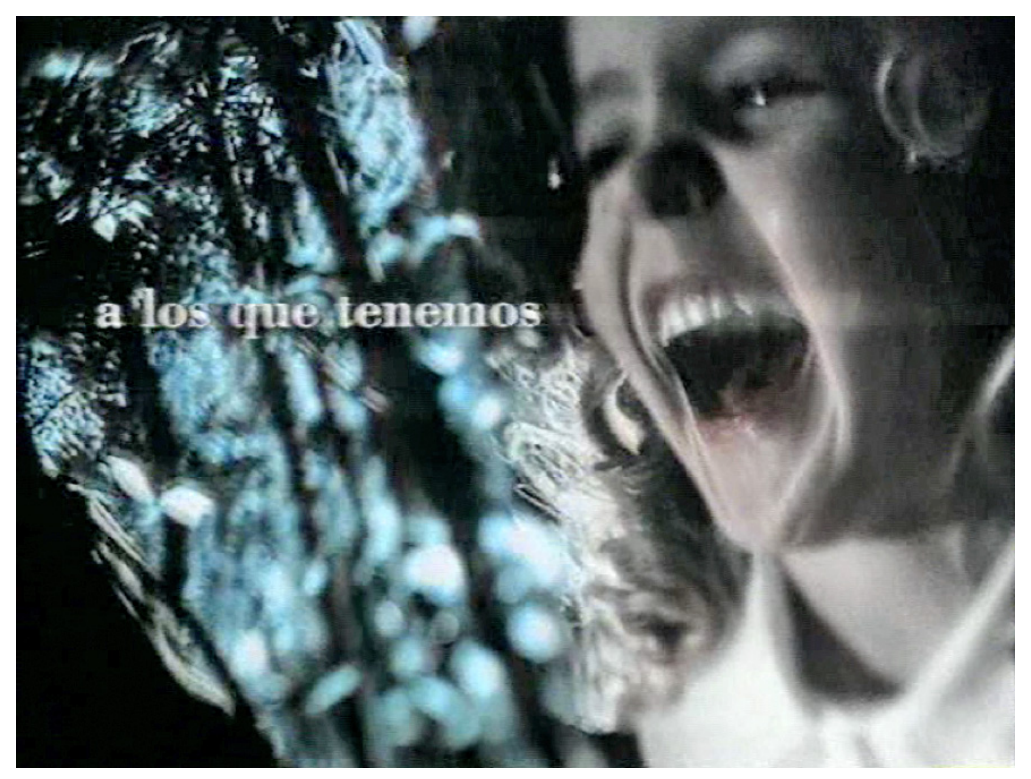

Fig. $n^{\circ}$ 4. Componente estésico. El sonido y el tacto. Movistar (Telefónica, 1996)

Junto a la manifestación visual somática nos encontramos la acción lingüística de un narrador en off que introduce a través de la verbalidad otros sentimientos como son: el perdón, la aceptación y la negación. Además, incorpora el tema del amor cuya imagen tendrá lugar tanto en soledad como en compañía de la pareja (frente al resto de sensaciones que se producen en la soledad del sujeto del relato).

Además de la manifestación somática de estos sentimientos, el creativo recurre en esta etapa del signo Movistar a un sistema semisimbólico para fijar su identidad significativa.

\subsection{El semisimbolismo como expresión significativa}

A este respecto, Floch nos habla de sistema "semi-simbólico" para diferenciar el sistema simbólico del sistema semiótico en función a la relación de conformidad entre el plano del contenido y el plano de la expresión, siendo esta relación total en el sistema simbólico e inexistente en el semiótico. Floch (1990: 130-131) se refiere al sistema "semi-simbólico" como otro tipo de relación:

¿Por qué hablar de sistema semi-simbólico? Para distinguir este tipo de sistema de otros dos definidos por L. Hjelmslev: los sistemas simbólicos son los sistemas semióticos propiamente dichos. Los sistemas simbólicos son el lenguaje en sus dos planos-el plano de la expresión y el plano del contenido- en conformidad total; a cada elemento de la expresión le corresponde uno y solo un elemento del contenido, hasta tal punto que no sirve distinguir ahora entre los dos planos porque presentan la misma forma [...] El sistema semiótico propiamente dicho es el lenguaje donde no hay conformidad entre los dos planos, donde se necesita distinguir -y estudiar separadamente- expresión y contenido [...] 
Si se asume que el sistema simbólico constituye un tercer tipo es porque depende de otra forma de semiosis, de otra relación entre expresión y contenido.

Siguiendo los postulados de Floch, hablaremos de la existencia de un sistema "semisimbolico" como estrategia identificativa de marca analizando el significado conjunto, del plano de la expresión y del plano del contenido. Para ello, nos detendremos en el mismo spot. Como señalamos arriba, en este anuncio el narrador en off nos enuncia lo siguiente:

"¿Por qué tenemos dos oídos? Uno para escuchar a los que tenemos cerca. ¿̇ el otro? El otro para escuchar a los que tenemos lejos".

De este texto se extraen semánticamente los siguientes conceptos:

- La escucha

- La soledad

- La pareja

- La cercanía

- La lejanía

- La posesión

Posteriormente nos encontramos con la narración visual de estas categorías ¿̇Cómo se representan visualmente estos conceptos? Para responder este interrogante, acudimos al spot seleccionado.

En primer lugar, nos encontramos con el concepto de la escucha representado de la siguiente forma:

- Trazo lateral/trazo frontal. La lateralidad facial del actante sujeto es una constante en estos anuncios. La mayoría de personajes se presentan en escena sin mirarnos, atentos a otra realidad ausente para el co-enunciatario.

- Identificación metonímica del órgano capaz de realizar dicha acción: el oído. Asistimos a un "viaje hacia el interior del oído".

El actante sujeto muestra su lateralidad en la que la oreja centraliza la imagen hasta el punto de que su morfología se identifica metafóricamente con la de tres letras en mayúsculas localizadas sobre ella: la "D", la "O" y la "S". ¿Qué tienen en común la linealidad del trazo de estas letras con la de la oreja? La apertura, la circularidad y la verticalidad. Tres cualidades identificativas del discurso de marca instaladas en la acción de escuchar. 
Más adelante nos topamos con la presencia de otro actante sujeto que nos muestra su lateralidad, aunque esta vez desde el ángulo derecho, el cual nos va a ilustrar la acción de escuchar en una aproximación a tempo lento hacia el interior del oído para instalarnos en los personajes, en su cercanía. Observemos cómo el sonido cercano es representado tanto lingüísticamente mediante la palabra difuminada "cerca", como linealmente en horizontal aunque ad avanti de lo que el co-enunciatario desconoce.

- Apertura. Dicha categoría la encontramos en la figura de la boca abierta de una niña que está gritando en silencio al tiempo que su boca se torna en el centro de la imagen en un primer plano lateral derecho.

- Ausencia de sonido traducible en una soledad de interacción, en la ausencia de discurso, de contacto.

Dicha ausencia nos introduce en el segundo concepto de este anuncio; la soledad. Veamos cómo aparece:

- Ausencia de interacción, como hemos visto, la cual se corresponde lingüísticamente con el determinante numeral cardinal "uno" representado en:

1. Una única oreja

2. La lateralidad

3. Un único actante

4. El término uno

- Ausencia de pluralidad representada espacialmente, pues nos encontramos en espacios en los que el actante sujeto experimenta la unicidad. En estos casos el actante sujeto sólo se siente acompañado por los elementos de la naturaleza, como ejemplifica la acentuada presencia del agua y de las hojas de los árboles en movimiento constante alrededor de los personajes.

- Trazo difuminado/ trazo nítido. La ausencia de compañía humana del actante sujeto se manifiesta mediante esta oposición categórica. En el spot nos encontramos con que tres miembros del cuerpo aparecen difuminados: la cabeza y la mano izquierda del personaje masculino, así como las piernas del femenino. La nitidez se aprecia, especialmente, en la cara del personaje femenino que aparece en la escena mirando hacia su lado izquierdo, aunque no hacia la presencia del personaje masculino. La mirada del actante femenino es triste, desolada, encerrada, negra; una mirada que se funde con la oscuridad cromática ambiental y con el lugar en el que se desarrolla la acción: el interior del hogar. Como puede verse, la única presencia, además de la humana, es la de un sillón de forja para dos ocupado, únicamente, por la actante femenina. La verticalidad no deja de presentarse en la escena; esta vez la encontramos en la forma de la silla y en la posición del actante masculino. 
- La difuminación, la discontinuidad y la presencia natural introducen a los personajes en un ambiente de misterio mágico en el que rezuman sentimientos de personajes diversos: de niños, de hombres y de mujeres. Todos están solos y todos están sintiendo.

Dicha soledad es experimentada como consecuencia de la lejanía la cual se representa en escena mediante:

- La huella del camino. Nos encontramos a unos personajes que están caminando hacia delante cuyas huellas reflejan el indicio de verticalidad de la acción. La lejanía del personaje es proyectada a través del plano largo en espacios naturales. En el spot observamos la conjunción de dos categorías: claridad/ oscuridad del actante sujeto que realiza la acción. La verticalidad oscura del mismo contrasta con la claridad del espacio natural en el que se está efectuando la acción. Fijémonos en la presencia del sol como signo del mensaje de marca cuyo resplandor adopta la imagen de estrella. Sin embargo, el movimiento es lo que une a ambos. Todo está vivo, todo fluye, todo está haciéndose. La naturaleza es el único compañero del ser humano. Dicha naturaleza fluctúa entre la montaña y el mar o el mar y la arena adoptando siempre una presentación vertical con respecto al sujeto.

El viaje hacia el centro de los sentimientos nos conduce a los deseos más íntimos el sujeto sintiente como son el amor y el deseo de cercanía, los cuales aparecen del siguiente modo:

- A través del determinante/ pronombre numeral cardinal "dos", identificado catafóricamente con el distributivo "el otro". Estamos ante un deseo de otredad.

- Por medio de la pareja. Los sujetos en el progreso de la acción se van juntando de dos en dos experimentando y expresando el placer del amor. Dicha unión se visualiza de diversas formas:

1. Unión de las manos. De nuevo movimiento y verticalidad alrededor de la circularidad y movimiento del agua.

2. Unión sentimental contigua. Nos encontramos con una imagen en la que aparece la cabeza de un actante femenino sobre un libro abierto cuyas hojas van pasando (apertura y movimiento).

3. Unión de los pies acariciándose en la arena de la playa.

- Mediante la fusión naturaleza y humanidad en la imagen en la que la niña acaricia un conejo mostrado también de forma fragmentada y lateral. La naturaleza forma parte de la niña, pues sobre su cabeza se elevan verticalmente dos orejas de conejo (verticalidad y movimiento).

Pasamos a esquematizar el sistema semisimbólico de la comunicación sentimental del modo siguiente: 
TABLA N 1. ESQUEMA DEL SISTEMA SEMISIMBÓLICO DE LA COMUNICACIÓN SENTIMENTAL

\begin{tabular}{|l|l|l|}
\hline EXPRESIÓN & $*$ Claridad & $*$ Oscuridad \\
& $*$ Difuminado & $*$ Contrastado \\
& $*$ Policromía & $*$ Monocromía \\
& $*$ Frontalidad & $*$ Lateralidad \\
& $*$ Pareja & $*$ Uogico \\
& $*$ Naturaleza & $*$ Disjunción objeto de \\
& $*$ Adjunción mensaje & de identidad y objeto de \\
& valor & \\
\hline CONTENIDO & $*$ Euforia & $*$ Disforia \\
& & \\
\hline
\end{tabular}

Nos encontramos, pues, en una etapa en la que lo característico es la valorización utópica, donde el sujeto se realiza en escena junto a su objeto de valor anhelado por él, pero ¿̇dónde está el objeto de valor en estos relatos?

Hasta el momento la narratividad nos ha ofrecido la visualidad de unos actantes solitarios o en parejas separados de su objeto de valor en la escena. No obstante, a pesar de que físicamente (hasta este momento) no sea visto por el coenunciatario sí está presente, en un mensaje de identidad creado a través de todas las estrategias pasionales y semisimbólicas ya analizadas, provocando que el personaje controle su disforia transformándola en euforia mediante la posesión y la cercanía de su objeto de valor.

\section{EL NOMBRE PROPIO PUBLICITARIO: UNA MARCA CON SIGNIFICADO}

Después del análisis de estos spots del signo Movistar, cabe preguntarse: $\dot{\imath} \mathrm{El}$ nombre propio publicitario es sólo una marca sin significado? ̇es simplemente una marca usada para permitir a los individuos devenir sujetos de discurso?

Klein afirma que la marca "es el significado esencial de la gran empresa moderna" (Klein, 2005: 33), "la personalidad de la empresa" (Klein, 2005: 34), productora de "sentimientos" e inculcadora del propio significado de las grandes empresas (Klein, 2005: 35), el "alma de la empresa", la inversión en "un valor puro", en un "valor de la empresa" (Klein, 2005: 36).

Como vemos, las marcas constituyen el significado de las empresas identificando su alma; la marca es un acto de creación de identidad significativa cuyo vehículo transmisor es la publicidad.

"Las marcas no son sólo una mascota o un gancho, ni una imagen impresa en las etiquetas de los productos" (Klein, 2005: 35), sino que, como señala Klein, a través de la 
marca las compañías en su totalidad pueden tener una identidad proyectando su imagen como "su camisa", llevando "la marca en el alma" (Klein, 2005: 35).

Las empresas se consideran "vendedoras de significado" (Klein, 2005: 48), donde la marca posee un componente espiritual y conceptual como experiencias y estilos de vida, de tal forma que, apunta Klein, el verdadero negocio es "crear una mitología corporativa lo suficientemente poderosa como para infundir significado a estos objetos brutos imponiéndoles su nombre" (Klein, 2005: 49).

La marca, pues, no es un producto, sino un significado (Klein, 2005: 51):

"Con la manía de las marcas ha aparecido una nueva especie de empresario que nos informa con orgullo de que la marca $X$ no es un producto sino un estilo de vida, una actitud, un conjunto de valores, una apariencia personal y una idea [...] Renzo Renoso, el propietario de Diesel Jeans, dijo a la revista Paper: "nosotros no vendemos un producto, vendemos un estilo de vida. Creo que hemos creado un movimiento [...]. El concepto Diesel está en todas partes. Es la manera de vivir, la manera de vestir: es la manera de hacer las cosas."

En relación a ello, Rey (1971: 126) afirma que, "se realiza un acto [el bautismo] y el esto [el objeto, el mundo] entra en el reino del significado". La denominación, pues, es esencial en la publicidad y es un soporte de predicado.

A este respecto, Péninou (1976: 95) afirma lo siguiente:

"La función publicitaria primordial, tanto por la cronología de sus esfuerzos como por la perennidad de sus resultados, apunta a la imposición de un Nombre. La publicidad, ante todo, es un gran baptisterio donde las producciones más dispares, salidas de progenitores innumerables, esperan el sello de su identidad [...] de esta agua bautismales surgió la Marca, cuyo nacimiento proclama a los cuatro vientos imponiendo su apelación y acompañándola en su destino en lo sucesivo."

Para Péninou, como dijimos, "el objetivo supremo es la conquista de los mercados mediante la autoridad exclusiva del Nombre Propio, cuyo extremo es la reducción del mercado de demanda a la demanda del Nombre" (Péninou, 1976: 96), de tal forma que se puede hablar de "la publicidad de los nombres propios" o "publicidad de marca" (Péninou, 1976: 95).

Pero, ¿̇qué se consigue a través de este acto de denominación? La identificación de una identidad, la "localización elemental de una individualidad" (Péninou, 1976: 97). A este respecto, Péninou (1976: 97) postula lo siguiente: 
"La identificación de un valor [...] el estatuto marcado de un bien le confiere un estado intermedio entre el de 'vrac' (el granel) (es decir, el desecho) y el de 'monstruo' (en el sentido de anomalía, de singularidad). Vrac designa en su origen un estado desordenado y desvalorizado de la materia [...] El monstruo absoluto sería aquel que, por su singularidad absoluta, no pudiera ponerse en relación con nada, pues escaparía a todo sistema de valores: sería lo Innombrable. El asignar a un bien un signo, una marca, no fija su valor con precisión, pero autoriza la oscilación de éste en el interior del campo de variación delimitado por las condiciones inferior y superior, con lo que el valor resulta entonces indefinido."

La denominación de un producto, supone la identificación de un significado, de un valor relativo, el paso de lo innombrado a lo nombrado y, además, como señala Péninou (1976: 97), "el paso del realismo de la Materia (el nombre común) al simbolismo de la Persona (el nombre propio), de tal forma que la marca se asimila a una persona introduciendo el objeto en todo un circuito personal, haciéndola "beneficiaria de una promoción fantástica" (Péninou, 1976: 97).

Por tanto, la denominación de un objeto supone un existir, un soporte de identidad, "un predicado", un "soporte del Ser" (Péninou, 1976: 100) en el que, además, se produce una "predicación", un aporte de valor mediante lo que Péninou (1976: 100) denomina "publicidad del Atributo", heredando un carácter propio:

"...La marca heredará una psicología y se incorporará a una historia. Tendrá derecho a rasgos de carácter (la "personalidad" de la marca) que salvaguardan su individualidad e impidan su reabsorción en el colectivo anónimo. Se la convidará a participar en el intercambio de los hombres, como agente de la imagen que se les propone ambicionar o a cuya entrega se les convence; entrará en su patrimonio, participará en sus trabajos cotidianos, en el embellecimiento de los cuerpos, en la calidad de la vida."

A través del nombre, los objetos reciben la vida; una vida cargada de significado y caracterizada por dos atributos: lo efímero y lo distinto.

Al tener existencia y ser análogos a la persona, las marcas poseen límites, con una historia "en perpetuo movimiento" (Péninou, 1976: 100-101).

El nombre de marca no es en sí mismo más que una promesa sobre la cual no puede construirse nada duradero y que no se sitúa en el corazón mismo de una red de asociaciones, lo más discriminatorias posibles, que añadan a su identidad una personalidad. 
Por tanto, para garantizar una duración de la personalidad de la marca en la memoria colectiva, la denominación se convierte en el primer paso a fin de distinguir una esencia única subjetiva (personalizada) cargada de significado, de intensión léxica. En conclusión, la marca es un signo.

\section{CONCLUSIONES}

Desde una perspectiva filosófico-lingüística el nombre propio es una marca sin significado con la única función de identificar pero sin describir, sin significar, sin connotar. Todos estos argumentos carecen de justificación ante la marca publicitaria donde el nombre propio se constituye en la esencia de la empresa, en una máquina cargada de significado.

Concretamente, en el presente artículo hemos analizado el significado de la marca Movistar que, lejos de ser un objeto-producto práctico, útil y material, es un sujeto hacedor con personalidad propia, un ser vivo, un signo.

El año 1996 se caracteriza por un estado latente de competitividad en telecomunicaciones, por tanto, las empresas necesitan fijar su alma, su identidad y esto se consigue con una marca que vivirá junto con su significado, con su discurso. Es así, como nace la marca Movistar acompañada de una valorización utópica de deseo a través de la cual el sujeto realiza su propia identidad cuando se conjuga con su objeto de valor. El portador desea autorrealizarse a través de la posesión de este signo.

En conclusión uno de los objetivos de la publicidad es cifrar la conquista de los mercados mediante "la autoridad exclusiva del nombre propio"; por tanto, las marcas comerciales requieren de una existencia lingüística para su funcionamiento. Con todo, y frente a los postulados de la lingüística, el estudio de la formación de una marca requiere de una orientación semiótica donde el nombre propio es una marca con significado, "una machina semiotica" (Semprini, 1993: 55-56) esencial de la personalidad de la gran empresa moderna.

\section{BIBLIOGRAFÍA}

DEL RíO LANZA, A.; VÁZQUEZ CASIELLES, R.; IGLESIAS ARGÜELLES, V. "El valor de marca: perspectivas de análisis y criterios de estimación". Cuadernos de gestión, 2, 2002, pp. 87102.

FABBRI, P. La svolta semiótica, Roma-Bari, Laterza, 1998.

FERNÁNDEZ MORENO, L. La referencia de los nombres propios, Trotta, Madrid, 2006.

FREGE, G. "Funktion und Begriff", Funktion, Begriff, Bedeutung, 1, 1891, pp. 17-39, 
_."Ubre Sinn und Bedeutung", Zeitschrift für Philosophie und philosophische Kritik, 100, 1891, pp. 25-50.

_."Der Gedanke", Beiträge zur Philosophie des deutschen Idealismus, 2, 1891, pp. 5877.

_Wissenschaftlicher Briefwechsel, Felix Meiner, Hamburgo, 1976.

_Nachgelassente Schriften, Felix Meiner, Hamburgo, 1983.

_Ensayos de semántica y filosofía de la lógica, Tecnos, Madrid, 1998.

FLOCH, J. M. Semiotica, márketing e comunicazione. Dietro i segni, le estrategia, Milano, Franco Angeli, 2002.

GARCÍA GALLARÍN, C. El nombre propio. Estudios de historia lingüística española, Patrom, 1999.

GREIMAS, A. J. Sémantique structurale, Paris, Larousse, 1966.

JONASSON, K. Le nom propre. Constructions et interpretations, Duculot, Louvain-la-Neuve, 1994.

KAPLAN, D. "Bob and Carol and Ted and Alice", Approaches to Natural Language, 1, 1973, pp. 490-518.

KLEIN, N. No Logo. El poder de las marcas, Paidós, Barcelona, 2005.

KRIPKE, S. "Identity and necessity", Identity and Individuation, 2, 1971, pp. 135-164.

_"Speaker's reference and semantic reference", Contemporary Perspectives in the

Philosophy of Language (Midwest Studies in Philosophy), II, 1977, pp. 255-276.

__Naming and Necessity, Blackwell, Oxford, 1980.

MARRONE, G. C'era una volta il telefonino. Un'indagine sociosemiotica, Roma, Meltemi, 1999.

MILL, JOHN. S. A System of Logic Ratiocinative and Inductive. Being a Connected View of the Principles of Evidence and the Methods of Scientific Investigation, University of Toronto Press, Toronto, 1843.

PÉNINOU, G. Semiótica de la publicidad, Barcelona, Gustavo Gili, Barcelona, 1976.

PÉREZ YUSTE, A. "El Proceso de Implantación de la telefonía móvil en España", Antena de Telecomunicación, 153, 2002, pp. 26-31.

REY, A. "La Conscience du poète", Literature, 4, 1971, pp. 126.

SÁNCHEZ CORRAL, L. "El nombre propio como imagen semiótica del referente", E.L.U.A., 6, 1990, pp. 207-227. 
SEMPRINI, A. Marche e mondi possibili. Un approccio semiotico al marketing della marca, Tipomonza, Milano, 1993.

ULLMANN, S. Semántica. Introducción a la ciencia del significado, Madrid: Taurus, Madrid, 1991. 